\title{
Variable-Flavor-Number Scheme in Analysis of Heavy-Quark Electro-Production Data
}

\author{
S. Alekhin ${ }^{1}$, J. Blümlein ${ }^{2}$, S. Klein ${ }^{2}$, and S. Moch ${ }^{2}$ \\ 1- Institute for High Energy Physics \\ Pobeda 1, 142281 Protvino, Moscow Region - Russia \\ 2- Deutsches Elektronensynchrotron DESY \\ Platanenallee 6, D-15738 Zeuthen - Germany
}

\begin{abstract}
We check the impact of the factorization scheme employed in the calculation of the heavy-quark deep-inelastic scattering (DIS) electro-production on the PDFs determined in the NNLO QCD analysis of the world inclusive neutral-current DIS data combined with the ones on the neutrino-nucleon DIS di-muon production and the fixed-target Drell-Yan process. The charm-quark DIS contribution is calculated in the generalmass variable-flavor-number (GMVFN) scheme: At asymptotically large values of the momentum transfer $Q$ it is given by the zero-mass 4 -flavor scheme and at the value of $Q$ equal to the charm-quark mass it is smoothly matched with the 3-flavor scheme using the Buza-Matiounine-Smith-van Neerven prescription. The PDFs obtained in this variant of the fit are very similar to the ones obtained in the fit with a 3 -flavor scheme employed. Our 5-flavor PDFs derived from the 3-flavor ones using the NNLO matching conditions are used to calculate the rates of $W^{ \pm} / Z$ and $t \bar{t}$ production at the Tevatron collider and the LHC at NNLO.
\end{abstract}

1. A clear theoretical understanding of the heavy-quark electro-production is necessary for the phenomenology of lepton-nucleon DIS at HERA since it contributes up to $25 \%$ to the DIS inclusive cross section at small $x$ 2, 3. This is particularly important for the validation of the parton distribution functions (PDFs) in the kinematic region relevant for the foreseen experiments at the LHC since, despite the impact of collider data on the PDFs, DIS remains an unique source of information about PDFs at small $x$. Two complementary approaches were suggested to calculate the DIS heavy-quark contribution. One of them is based on the factorization scheme with three light quarks in the initial state. In the 3-flavor scheme the heavy-quark production is calculated in fixed order of perturbative QCD with complete corrections up to the $O\left(\alpha_{s}^{2}\right)$ and partial corrections up to $O\left(\alpha_{s}^{3}\right)$ available [4, 5, 6, 17. The logarithmic terms in the QCD corrections rise in the asymptotic region of the momentum transferred $Q \gg m_{h}$, where $m_{h}$ is the heavy quark mass. Therefore they must be re-summed for such kinematics 8 . This leads to the concept of the variable-flavor scheme (VFN) with the heavy quarks considered in the same way as the light-flavor ones. In the VFN scheme large-log re-summation suggested in Ref. 8 is performed through the QCD evolution of the heavy-quark PDFs [9]. The VFN scheme is valid only at asymptotically large values of $Q$ and cannot be routinely extrapolated to the low- $Q$ region. For realistic kinematics it has to be extended to the case of a general-mass VFN (GMVFN) scheme, which, at small $Q$, must give a smooth matching with the 3-flavor scheme. Different variants of the GMVFN scheme are used in the context of the global fit of PDFs based on small- $x$ DIS data [10. At the matching point $Q=m_{h}$ the structure function $F_{2}^{h}$ calculated within the GMVFN schemes of Ref. 10] is continuous. However these schemes do not guarantee a smooth transition of 

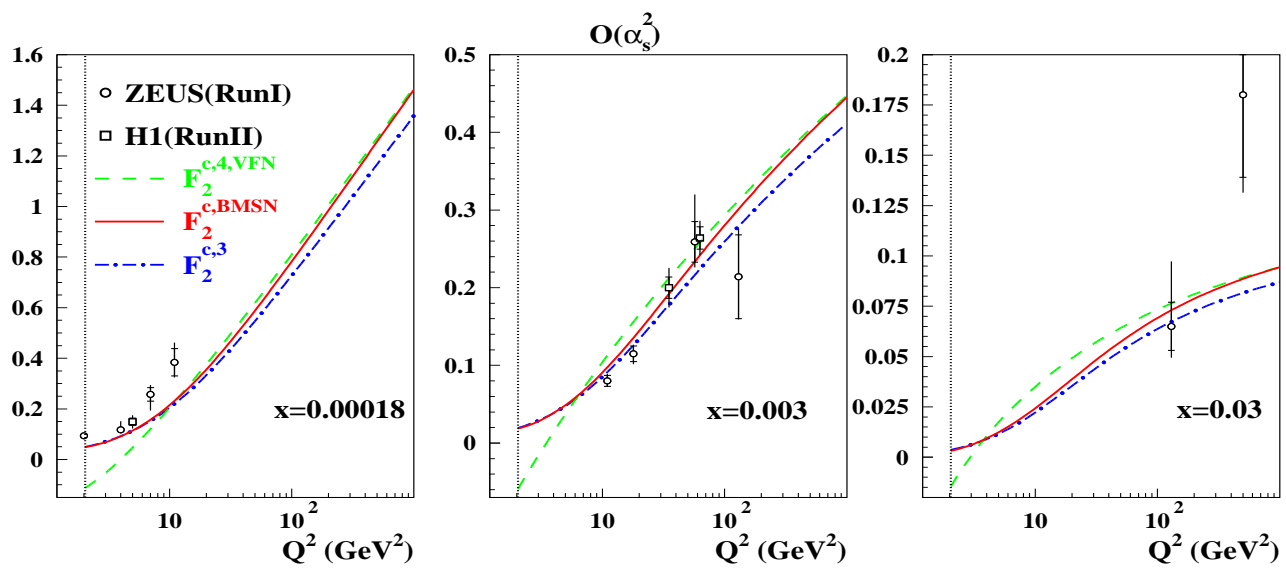

Figure 1: The values of $F_{2}^{c}\left(x, Q^{2}\right)$ calculated in the different schemes compared to the data by H1 2] (squares) and ZEUS [3] (circles) collaborations (dotted-dashes: the 3-flavor scheme; solid lines: the BMSN prescription of the GMVFN scheme, dashes: the massless 4-flavor scheme).

$F_{2}^{h}$ between the 3 -flavor and the VFN schemes. In our study we consider a variant of the GMVFN scheme based on the Buza-Matiounine-Smith-van Neerven (BMSN) prescription of Ref. [1], which indeed provides a smooth behavior of $F_{2}^{h}$ at $Q=m_{h}$, and perform a phenomenological comparison of this prescription with the 3 -flavor scheme in the PDFs fit.

2. The conclusion about the impact of the scheme evidently depends on the kinematic coverage and precision of the data used in the fit. Figure 1 shows a comparison of the charm structure function $F_{2}^{c}$ calculated in the 3-flavor scheme and the BMSN prescription of the GMVFN scheme with the use of the MRST2001 PDFs [12] to the representative set of the recent HERA collider data by $\mathrm{H} 1$ and ZEUS [2, 3]. The available data on $F_{2}^{c}$ are not sensitive to the difference between the 3-flavor and the GMVFN scheme in the BMSN prescription. The best sensitivity of the data to the scheme choice appears at $x \sim 0.003$, however even in this case the errors in the data are quite big as compared to the scheme differences. This does not look like a specific feature of the BMSN prescription since even at maximal values of $Q$ reached by the existing experiments the difference between the massless 4 -flavor scheme and the 3-flavor scheme is smaller than the data uncertainties. In particular this happens because the NLO corrections of Ref. 5 to the heavy-quark electro-production greatly reduce the need of large-log re-summation 13. With the NNLO corrections of Ref. [7 this need should be further reduced. At small $Q$ the difference between the 3-flavor and BMSN scheme is suppressed, similarly to any other GMVFN scheme, which provides smooth matching with the former. The discrepancy between theory and data at small $Q$ and $x$ observed in Figure 1 cannot be resolved by a scheme change. It rather can be cured by the partial NNLO corrections of Ref. 14 due to the soft gluon re-summation near threshold.

3. The inclusive DIS data are more sensitive to the choice between the VFN and 3-flavor scheme because of higher accuracy. However, the PDFs obtained from the fits based on these two variants are still similar. We check this point for the example of a combined fit of PDFs to the inclusive DIS data supplemented by the fixed-target Drell-Yan data and the DIS di-muon production data, which improves the flavor separation of the quark PDFs. 


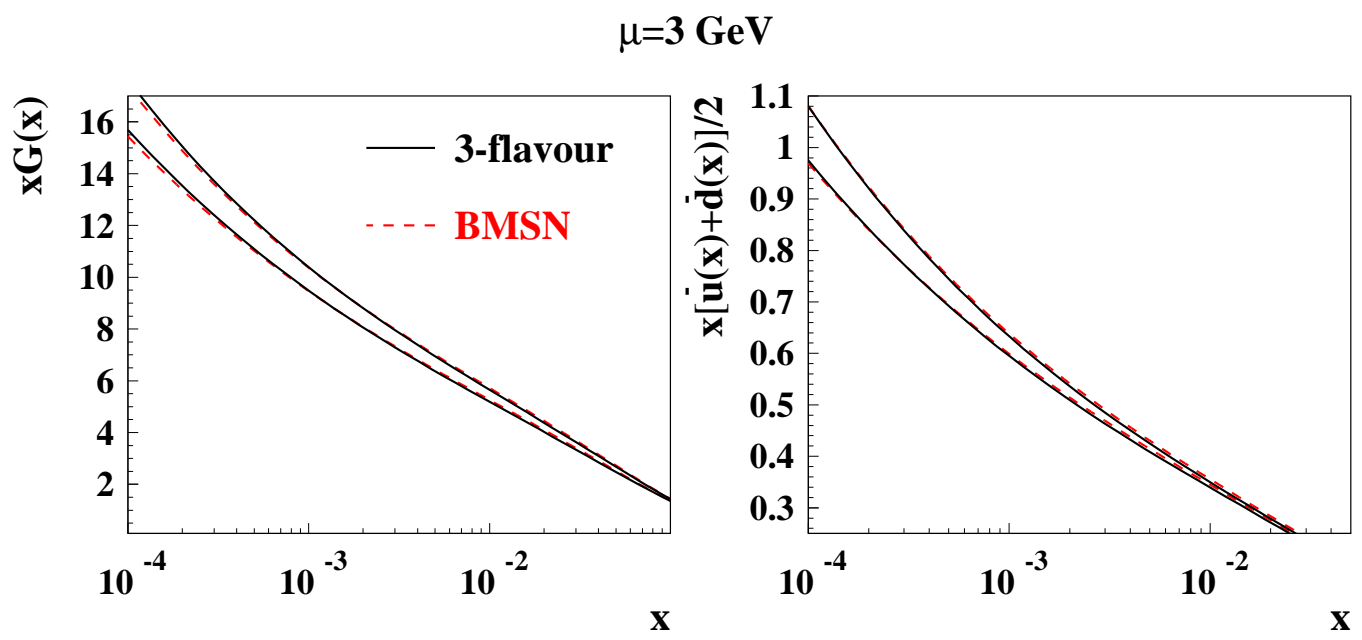

Figure 2: The gluon (left panel) and sea (right panel) distributions at the factorization scale of $\mu=3 \mathrm{GeV}$ obtained in two variants of the fit. Solid lines: 3 -flavor scheme, dashed lines : GMVFN scheme in the BMSN prescription.

We consider two variants of the fit, with $F_{2}^{c}$ calculated in the 3-flavor scheme or in the BMSN prescription. The NNLO corrections to the PDF evolution [15], the light-parton coefficient functions [16], and the NLO corrections of Ref. [5] to the heavy-quark electroproduction coefficient functions are taken into account. The non-QCD corrections applied and other details of the fit can be found elsewhere 17. The gluon and sea distributions obtained in the two variants of the fit are compared in Figure 2. The difference between the two variants of the fit is marginal and is in agreement with the comparison given in Figure 1, In the case of Thorne-Roberts (TR) prescription of Ref. [18] employed for the GMVFN scheme the impact of the scheme choice is somewhat bigger [19. That might be related to a kink in $F_{2}^{c}$ at $Q=m_{c}$ for small $x$. For the ACOT prescription 9 and the $\operatorname{ACOT}(\chi)$ modification of this prescription [20] employed in the NNLO analysis of Ref. 21] this kink is more pronounced than for the case of the TR prescription. As a result, the heavyquark contribution to the inclusive structure function is overestimated and the light-parton distributions are underestimated, correspondingly.

4. Contrary to the case of DIS for many processes of interest at the energies of the Tevatron collider and the LHC the use of a VFN scheme is justified since the typical factorization scale is much bigger than the $c$ - and $b$-quark masses. Moreover, very often such an approach is very efficient since it allows to simplify matrix element calculations. For the purposes of collider phenomenology applications we generate the NNLO 4-flavor PDFs using the matching conditions of Ref. [1] with the 3-flavor PDFs obtained in our fit as input. Similarly, we use these 4-flavor PDFs to produce the 5-flavor ones. Figure 3 shows a comparison of our 4-flavor PDFs at the scale of $\mu=2 \mathrm{GeV}$ with the NNLO MSTW08 PDFs of Ref. 21. at this scale the sea and gluon distributions by MSTW are smaller

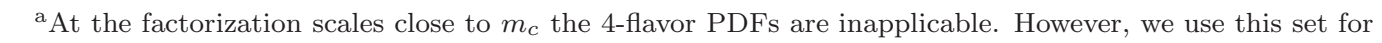
the purpose of comparison since the 3-flavor PDFs are unavailable for the MSTW08 case. 

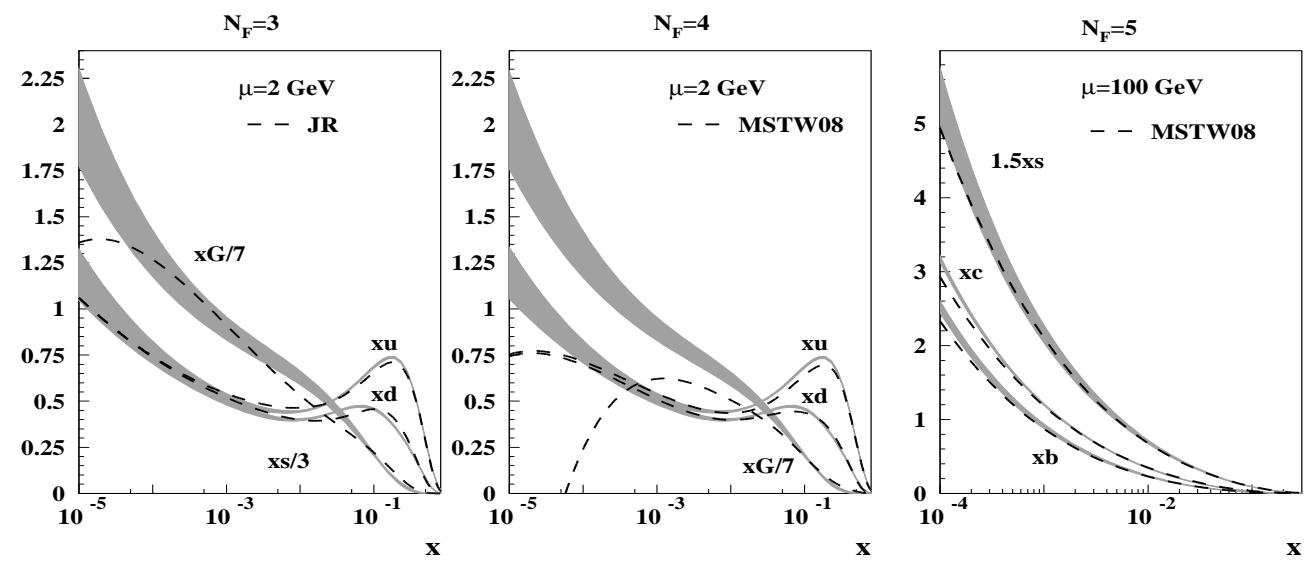

Figure 3: The $1 \sigma$ band for the representative set of our PDFs (shaded area) and the central values of other NNLO PDFs sets (dashes). Left panel: the 3-flavor $u$-, $d$-quarks and gluons at the scale of $\mu=2 \mathrm{GeV}$ compared to ones of the JR set; central panel: the 4-flavor $u$-, $d$-quarks and gluons at the scale of $\mu=2 \mathrm{GeV}$ compared to ones of the MSTW08 set; right panel: the 5 -flavor $s_{-}, c_{-}$, and $b$-quarks at the scale of $\mu=100 \mathrm{GeV}$ compared to ones of the MSTW08 set.

than ours, moreover the MSTW gluon distributions are negative at $x \lesssim 0.0005$. The difference in the gluon distribution of these two sets is sensitive to the recent measurement of the structure function $F_{L}$ by the H1 collaboration 23. The H1 data are in good agreement to the predictions of $F_{L}$ based on our PDFs, however, then go slightly above the MSTW08 ones and in that way prefer bigger gluons at small $x$. The agreement between our PDFs and the JR PDFs of Ref. 22 obtained in the fit performed in the 3-flavor scheme is much better (see Figure 3). In this

\begin{tabular}{|cc|c|c|c|}
\hline \multicolumn{2}{|c|}{$\sqrt{s}(\mathrm{TeV})$} & $\sigma\left(W^{ \pm}\right)[n b]$ & $\sigma(Z)[n b]$ & $\sigma(t \bar{t})[p b]$ \\
\hline 1.96 & $(\bar{p} p)$ & $26.2 \pm 0.3$ & $7.73 \pm 0.08$ & $6.91 \pm 0.17$ \\
\hline 7 & $(p p)$ & $98.8 \pm 1.5$ & $28.6 \pm 0.5$ & $131.3 \pm 7.5$ \\
\hline 10 & $(p p)$ & $145.6 \pm 2.4$ & $42.7 \pm 0.7$ & $342 \pm 15$ \\
\hline 14 & $(p p)$ & $207.4 \pm 3.7$ & $61.4 \pm 1.1$ & $780 \pm 28$ \\
\hline
\end{tabular}
case the gluon distributions differ only at $x \sim 10^{-5}$, in a region not being constrained by data. Our 5 -flavor $c-$ and $b$-quark distributions are larger than the MSTW ones. This is related to the difference in the gluon distribu-

Table 1: The integral $W^{ \pm} / Z$ and $t \bar{t}$ production cross sections at the energies of Tevatron and LHC with the $1 \sigma$ uncertainties estimated from the fit results in the present analysis. $\pm 0.5 \mathrm{GeV}$, respectively, into the PDF uncertainties. For the case of $c-$ and $b$-quark PDFs the uncertainties in the masses are dominant sources. The value of the strong coupling $\alpha_{\mathrm{s}}\left(M_{\mathrm{Z}}\right)=0.1135 \pm 0.0014$ is obtained in our fit, in good agreement with the results of Ref. 22 and earlier results of Refs. 24, 17].

5. The rates of the $W^{ \pm} / Z$ and $t \bar{t}$ production at the Tevatron collider and the LHC obtained with our 5-flavor PDFs are given in Table 1 . The $W^{ \pm} / Z$ rates are calculated with the QCD corrections up to NNLO [25. In the case of the $t \bar{t}$ production only partial 
NNLO corrections of Ref. 26] stemming from the threshold gluon re-summation are taken into account. The uncertainties in the $W^{ \pm} / Z$ rates due to the PDFs vary from $1 \%$ at the Tevatron energy to $2 \%$ at the maximal LHC energy. Such a level of accuracy allows to use these processes as a luminosity monitor. Our estimate of the $t \bar{t}$ production rate for Tevatron is in agreement to the estimate obtained with the MSTW08 PDFs, while at the LHC energies the latter are bigger than ours. As in the case of $F_{L}$ this difference is related to the difference in the gluon distributions. Therefore $t \bar{t}$ production at the LHC can be used to discriminate between these two PDFs sets.

Acknowledgments. This work was supported in part by DFG Sonderforschungsbereich Transregio 9, Computergestützte Theoretische Teilchenphysik, the RFBR grant 08-02-91024, Studienstiftung des Deutschen Volkes, the European Commission MRTN HEPTOOLS under Contract No. MRTN-CT-2006-035505.

\section{References}

[1] Slides: http://indico. cern. ch/contributionDisplay $\cdot$ py? contribId=158\&sess ion Id=5\& conf $I d=53294$

[2] H1 Collaboration, F.D. Aaron, et al., arXiv:0907.2643 [hep-ex].

[3] ZEUS collaboration, S. Chekanov et al., Phys. Rev. D69 (2004) 012004.

[4] E. Witten, Nucl. Phys. B 104 (1976) 445;

[5] E. Laenen, S. Riemersma, J. Smith and W. L. van Neerven, Nucl. Phys. B 392 (1993) 162;

[6] E. Laenen and S. O. Moch, Phys. Rev. D 59 (1999) 034027.

[7] I. Bierenbaum, J. Blümlein and S. Klein, Nucl. Phys. B 820 (2009) 417.

[8] M. A. Shifman, A. I. Vainshtein and V. I. Zakharov, Nucl. Phys. B 136 (1978) 157 [Yad. Fiz. 27 (1978) 455].

[9] M. A. G. Aivazis, J. C. Collins, F. I. Olness and W. K. Tung, Phys. Rev. D 50 (1994) 3102

[10] R.S. Thorne and W.K. Tung in : arXiv:0903.3861 [hep-ph].

[11] M. Buza, Y. Matiounine, J. Smith and W. L. van Neerven, Eur. Phys. J. C 1 (1998) 301.

[12] A. D. Martin, R. G. Roberts, W. J. Stirling and R. S. Thorne, Eur. Phys. J. C 28 (2003) 455.

[13] M. Glück, E. Reya and M. Stratmann, Nucl. Phys. B422 (1994) 37.

[14] S. I. Alekhin and S. Moch, Phys. Lett. B 672 (2009) 166.

[15] S. Moch, J. A. M. Vermaseren and A. Vogt, Nucl. Phys. B 688 (2004) 101; A. Vogt, S. Moch and J. A. M. Vermaseren, Nucl. Phys. B 691 (2004) 129.

[16] E. B. Zijlstra and W. L. van Neerven, Nucl. Phys. B 383 (1992) 525.

[17] S. Alekhin, J. Blümlein, S. Klein and S. Moch, arXiv:0908.2766 [hep-ph]; S. I. Alekhin, S. Kulagin and R. Petti, Phys. Lett. B 675 (2009) 433; S. I. Alekhin, K. Melnikov and F. Petriello, Phys. Rev. D 74 (2006) 054033; S. I. Alekhin, Phys.Rev. D68 (2003), 014002.

[18] R. S. Thorne and R. G. Roberts, Phys. Lett. B 421 (1998) 303.

[19] A. M. Cooper-Sarkar, arXiv:0709.0191 [hep-ph].

[20] W.K. Tung, S. Kretzer and C. Schmidt, J. Phys. G28 (2002) 983.

[21] A. D. Martin, W. J. Stirling, R. S. Thorne and G. Watt, arXiv:0901.0002 [hep-ph].

[22] P. Jimenez-Delgado and E. Reya, Phys. Rev. D 79 (2009) 074023.

[23] H1 collaboration, New measurement of $F_{L}$ at low $Q^{2}$ from H1, 17th International Workshop on DeepInelastic Scattering and Related Subjects Madrid, Spain (2009).

[24] J. Blümlein, H. Böttcher and A. Guffanti, Nucl. Phys. B 774 (2007) 182.

[25] R. Hamberg, W. L. van Neerven and T. Matsuura, Nucl. Phys. B 359 (1991) 343 [Erratum-ibid. B 644 (2002) 403]; R. V. Harlander and W. B. Kilgore, Phys. Rev. Lett. 88 (2002) 201801.

[26] S. Moch and P. Uwer, Phys. Rev. D 78 (2008) 034003; U. Langenfeld, S. Moch and P. Uwer, arXiv:0906.5273 [hep-ph]. 